\title{
Ultrasonic Thermometry Study and Portable Ultrasonic Thermometer Prototype
}

\author{
Lukáš Ondraczka \\ Department of Measurement \\ CTU in Prague - FEE \\ Prague, Czech Republic \\ ondralu3@fel.cvut.cz
}

\author{
Tomáš Ondraczka \\ Department of Cybernetics and Biomedical \\ Engineering \\ Technical University of Ostrava - FEI \\ Ostrava, Czech Republic \\ tomas.ondraczka@vsb.cz
}

\begin{abstract}
This paper presents an ultrasonic thermometry state of the art study and shows principle of the ultrasonic thermometry through experiments with an ultrasonic thermometer prototype. The study covers a brief ultrasonic temperature measurement research history and focuses on recent revelations in this field. The thermometer prototype is constructed to be portable and usable for educational presentations. Its principle, performance and ultrasonic temperature measurements results are described in the experimental part.
\end{abstract}

Keywords-ultrasonic thermometry; air temperature measurement; time of flight; ultrasonic thermometer;

\section{INTRODUCTION}

The ultrasonic thermometry is non-invasive, fast and precise method for measuring temperature. It is used in applications, where more common temperature measurement methods (e.g. mercury thermometer, thermocouple, thermistor, resistance temperature detector, infrared thermometer, optical fiber thermometer and many more) fail to provide acceptable results.

Principle of the ultrasonic thermometry is ultrasound velocity temperature dependence utilization. It is possible to find this dependence in any medium, generally the ultrasound velocity temperature dependence is linear in liquids, in gases the velocity rises with square root of temperature and in solids, the velocity falls with rising temperature.

The velocity is measured by the time of flight measurement between an ultrasonic transmitter and receiver at a fixed distance. Nowadays two basic methods are used, direct time of flight measurement and phase shift measurement. Time of flight measurement is simpler to apply, has wider measurement range and can be used for measurements over long distances (depending on the medium), but is more susceptible to signal amplitude variations caused by noise and therefore less precise. Phase shift method is more resistant to noise, shows higher precision, but its measuring range is limited by the wavelength of ultrasonic signal, since a phase ambiguity will occur when the temperature change is too high. In some papers the combination of both methods is successfully employed, with time of flight method measurement used for rough estimation of temperature and phase shift method for its refinement.
In this paper the time of flight method in an air medium is shown in the experiment, but the study deals with papers about ultrasonic distance or wind velocity measurements in various mediums and acoustic measurements using audible sound frequencies as well, since it is built on the principles related to the temperature measurements.

\section{STATE OF THE ART STUDY}

Ultrasonic temperature measurement is intensively studied scientific field nowadays, with rich history as well. This study reveals the papers which are related to this form of temperature measurement over the last 70 years. Focus is on up to date state of the art, but the most important works from the whole ultrasonic thermometry epoch are mentioned as well.

\section{A. First acoustic measurement systems}

First ultrasonic measurement systems were described in 1944 and first successfully constructed measurement system was completed in 1949 by Barett and Suomi [1], when they developed air temperature measurement system. A horizontal wind velocity measurement system was successfully developed by Schotland in 1955 [2]. First device able to measure the temperature and the wind speed simultaneously was made by Kaimal and Businger in 1962 [3] and [4].

\section{B. Atmospheric boundary layer behavior measurements}

Acoustic measurements are the best way to show the near surface atmosphere behavior. This fact led to very intense research in this area starting with Kaimal and Businger's work and persisting until cca 2010. A lot of studies were performed which mostly span around tomographic temperature and wind speed vector fields reconstruction with usage of time-offlight information between multiple transmitters and receivers.

In 1982 an ultrasonic anemometer/thermometer for field operation with microcontroller implemented was constructed by Hanafusa et al. [5].

The first work known to carry out the 2-D acoustic tomography successfully was done by D. Wilson and D. Thomson in 1994 [6]. Other progress was made by K. Arnold, A. Ziemann and A. Raabe [6-9] and S. Vecherin, V. Ostashev, A. Ziemann and D. Wilson [10-13]. 
This effort led to the development of a reliable and precise technique of the temperature and wind speed field reconstruction with usage of the acoustic time-offlight measurements.

Finally paper "Acoustic tomographic imaging of temperature and flow fields in air" by M. Barth and A. Raabe [14] has to be mentioned. This paper uses experience from previous work to present a 3D tomographic ultrasonic system for measurement of temperature and flow fields of air.

\section{Papers dealing with precise ultrasonic measurements}

In many papers, there is a need of very precise ultrasonic time-of-flight measurement to provide desired results. The ultrasonic thermometry is not an exception. Measurement precision is highly dependent on correct time-of-flight estimation, but this issue is present in another ultrasonic measurement applications as well. This chapter reveals papers which include this issue and are based on similar measurement techniques as the ultrasonic thermometry.

First fairly accurate work was done in 1997 [15] and deals with accurate ultrasonic distance measurement by combining time-of-flight and phase shift methods. Final accuracy was $\pm 1 \mathrm{~mm}$ on $1 \mathrm{~m}$ propagation path.

Very important in this field are papers from 2002 by S. Huang, C. Huang, K. Huang, M. Young and Y. Li. First deals with accurate distance measurement using binary frequency shift-keyed signal and phase detection [16]. Authors claim that accuracy of \pm 0.05 $\mathrm{mm}$ at a range of over $6000 \mathrm{~mm}$ was reached. Second paper released in the same year [17] deals with precise temperature measurement with ultrasound. Measurement distances from 6 to $10 \mathrm{~cm}$ were used and the best accuracy was found to be $\pm 0.05^{\circ} \mathrm{C}$ at $10 \mathrm{~cm}$ distance. This team also managed to build ultrasonic temperature measurement system in an infant incubator in 2003 [18]. In this practical implementation an accuracy of $\pm 0.2{ }^{\circ} \mathrm{C}$ on 1 meter propagation path was theoretically reached (only \pm 1 ${ }^{\circ} \mathrm{C}$ reference measurement was available).

Next team dealing with precise ultrasonic temperature measurement were scientists T. Liao, W. Tsai, C. Huang and H. Chen. Their first work (2004) uses ultrasonic measurement advantages for measuring temperature in automobiles, which can provide better temperature information for an air conditioner system than conventional thermistors [19]. It combined timeof-flight and phase shift methods and final accuracy of the system was $\pm 0.4{ }^{\circ} \mathrm{C}$ in dry environment (with higher errors assumed in environment where humidity changes). In 2005 this team developed measurement system with self-correction for humidity [20], with the same $\pm 0.4{ }^{\circ} \mathrm{C}$ accuracy as before but with resistance to errors caused by humidity changes. In 2006, this team developed high accuracy air temperature measurement system using MFCW (Multi-Frequency Continuous Wave) [21]. This paper dealt with measuring phase shifts and their differences on three ultrasonic frequencies, which highly enlarged phase shift measurement range and preserved its precision of \pm 0.4 ${ }^{\circ} \mathrm{C}$.
An ultrasonic measurement using MLS (Maximum Length Sequences)-modulated continuous waves was presented by Z. Huang, C. Qiao and Y. Wang in 2015 [22]. This work used MLS-modulated continuous waves to gain better resistance of the time-of-flight combined with the phase shift measurement against noise and obstructions in the vicinity of the measurement, which can cause multiple echoes. Using this technique an uncertainty of $40 \mu \mathrm{m}$ was achieved in distilled water on distances up to $200 \mathrm{~mm}$. This team implemented MLS-modulated measurement method on temperature measurement as well in 2016 [23]. Using the same setup as before, but with an extra mercury thermometer and thermostatic bath, measurement accuracy of $\pm 0.04{ }^{\circ} \mathrm{C}$ was achieved.

\section{Papers dealing with measurement in harsh environments}

Non-invasiveness is one of the ultrasonic measurement advantages. It allows measurements in applications where contact sensors would disturb the measurement too much and in applications where contact sensors would be destroyed or wouldn't perform well due to harsh environmental conditions. This chapter mentions the latter examples.

First example of ultrasonic application in harsh environments are measurements in nuclear reactors. Measured variable is temperature of over $2000{ }^{\circ} \mathrm{C}$. Thermocouples are restricted to $2000{ }^{\circ} \mathrm{C}$ and infrared thermometry is unable to measure an internal temperature and very sensitive to the interference from reacted gases. This area is studied in publications [24] and [25].

The measurement of the temperature and melt flow during polymer extrusion process is another interesting ultrasonic application. The temperature measurement of an extruderate is conducted in [26] and a melt flow measurement can be found in [27].

Some studies investigate material properties on higher temperatures by ultrasonic means. As an example a study of elastic properties of refractory materials [28] can be mentioned. This study examined the refractory materials properties in high temperatures (up to $1200^{\circ} \mathrm{C}$ ). Another study dealing with this issue investigates acoustic properties of materials for ultrasonic thermometry [29]. The sound propagation velocity and attenuation of ultrasonic waves in alumel, chromel and copel samples in wide temperature range $\left(0-1000{ }^{\circ} \mathrm{C}\right)$ were measured using ultrasonic measurements.

A study from 2014 deals with thermal measurements in a navy gun during firing from it [30]. The temperature was successfully measured by ultrasonic sensors (other sensors would be destroyed by the projectile or would disrupt an integrity of a gun barrel).

The last study from a measuring in the harsh environment category is "Ultrasonic $\mathrm{Al}_{2} \mathrm{O}_{3}$ Ceramic Thermometry in High-Temperature Oxidation Environment" from 2016 [31]. This study confirms ultrasonic thermometry as a feasible method for temperature measurements in oxidation environment with results in the range from $26^{\circ} \mathrm{C}$ to $1600{ }^{\circ} \mathrm{C}$. 


\section{E. Ultrasonic tomography studies}

Papers dealing with multi-dimensional temperature (sometimes simultaneously with wind flow) field measurement are mentioned in this chapter. A lot of studies related to this topic is already described in section above, but the studies here are not focused on the atmospheric boundary layer examination and usually conduct measurements in smaller areas or even different mediums than air.

First interesting paper is about a measurement of the temperature distribution in fluids using the ultrasonic tomography from 1994 [32].

Next work uses the ultrasonic measurement to estimate the temperature and wind fields [33]. The tomography is conducted with twelve ultrasonic transmitter and receiver pairs in a circle with one meter diameter.

The temperature and wind fields measurement is studied in the paper "Temperature Distribution and Wind vector Measurement Using Ultrasonic CT Based on Time of Flight Detection" [34] as well. This study uses 64 transmitter/receiver pairs for ultrasonic tomography measurements in air with heated aluminum base plate with two holes as a heat flow source. Measurement is conducted using the time-offlight method in combination with the phase shift measurement.

The last papers mentioned in this chapter were made by R. Jia, Q. Xiong, L. Wang, K. Wang, X. Shen, S. Liang and X. Shi in 2016 and 2017. This team studied ultrasonic thermometry in two papers so far. First one deals with one dimensional ultrasonic thermometry [35]. Time-of-flight measurement based on two signal envelopes comparison was used. Their second paper [36] extends the first one to two dimensional area, with the same measurement method implemented as in the first paper, but with more transducers (specifically four transmitters and eight receivers). The measurement area was square with $1 \mathrm{x}$ 1 meter dimensions and a heat source. Authors plan to implement this system in real industrial applications in further studies.

\section{F. Miscellaneous ultrasonic applications studies}

Special, unusual and creative ultrasonic measurement studies are mentioned here. All of them use the same base principles as the ultrasonic thermometry.

One of such studies is "Ultrasonic temperature measurement and its potential for food processing systems" [37]. This study present ultrasonic temperature measurement as a promising method in food industry.

Next area with good perspective in the ultrasonic measurement usage is a robot navigation. In a paper from 2013 [38] a group of scientists investigate a multiple ultrasonic echo detection to come up with satisfying method for corner or multiple objects detection.

A study about the ultrasonic thermometry inside tissues was conducted in 2015 [39]. This study implements the temperature measurements of tissues for the purpose of a diseases detection.

Last special paper deals with an ultrasonic guided wave tomography for ice detection [40]. It uses ultrasonic measurement method to detect ice on aircraft wings.

\section{THERMOMETER PROTOTYPE}

This part of the paper presents the ultrasonic thermometer prototype developed for the ultrasonic thermometry principle demonstration. It is constructed to be portable for convenient transfer possibility.

\section{A. Prototype parts}

The prototype consists of a measurement probe with an ultrasonic transducer and a fixed distance polypropylene wave guide made from a graduated cylinder, driving electronics (transmitter and receiver circuitry and STM32L4 microcontroller (MCU) on a development board), $12 \mathrm{~V}$ DC source and a PC with terminal software. The probe detail with electronics in background can be seen in the Fig. 1 .

\section{B. Transmitter and receiver circuit}

Transmitter and receiver circuits are both simple. The transmitter circuit uses a transistor as amplifier for the excitation signal from MCU (PWM signal with 40 $\mathrm{kHz}$ frequency and $50 \%$ duty cycle). It is supplied by $12 \mathrm{~V}$ DC source.

The receiver circuit consists of amplifier, LP Sallen-Key filter and comparator blocks. The amplifier amplifies the received signal to a reasonable level. The LP Sallen-Key filter circuit makes a signal envelope to prevent period ambiguity. The comparator circuit triggers an interrupt in $\mathrm{MCU}$ when the envelope reaches triggering voltage level set by resistor divider supplied from REF5050 precision voltage reference integrated circuit with $0.05 \%$ accuracy and $3 \mathrm{ppm} /{ }^{\circ} \mathrm{C}$ temperature drift. The REF5050 is also used as a $5 \mathrm{~V}$ supply in the receiver circuit. Transmitter and receiver schematics can be seen in the Fig. 2 and Fig. 3.

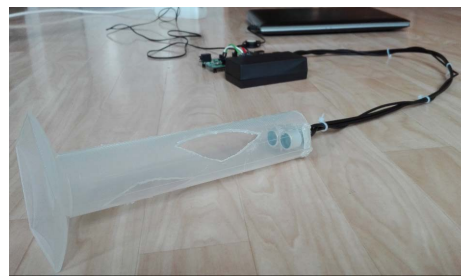

Figure 1. Ultrasonic thermometer prototype

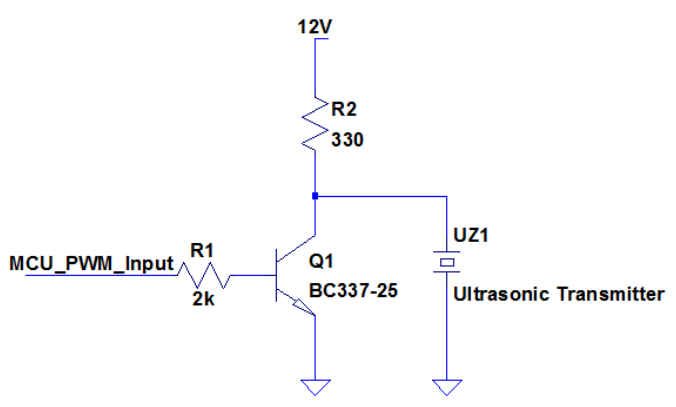

Figure 2. Transmitter schematics 


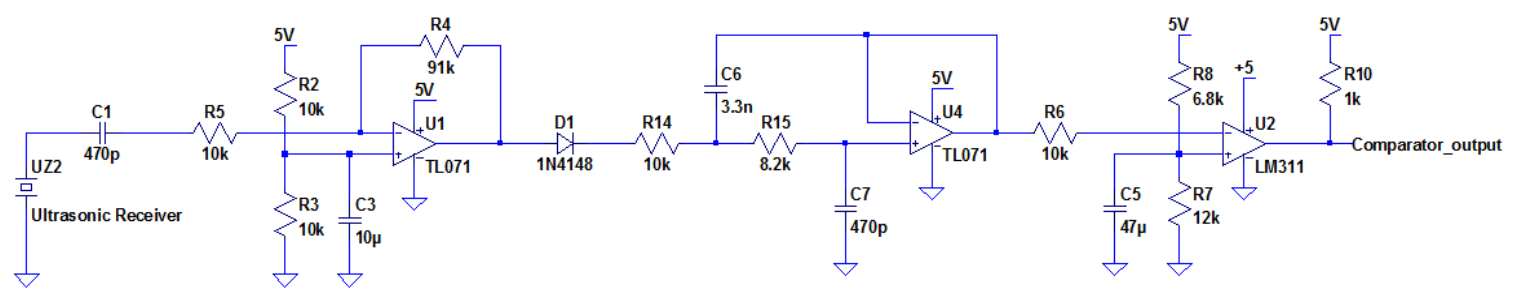

Figure 3. Receiver schematics

\section{Measurement procedure}

The measurement process in described in this chapter. Measurement starts with thirteen periods of exciting signal emission and reception of reflected echo by receiving transducer, as can be seen in the Fig. 4.

Next part is received signal amplification, rectification on diode and its filtering to make its envelope. The result can be observed in the Fig. 5 .

The envelope triggers comparator output when its voltage reaches $3.19 \mathrm{~V}$. This effect is shown in the Fig. 6.

Falling edge of the signal is detected by MCU and triggers the interrupt. Complete software flowchart is drawn in the Fig. 7. One measurement cycle takes 0.5 second.

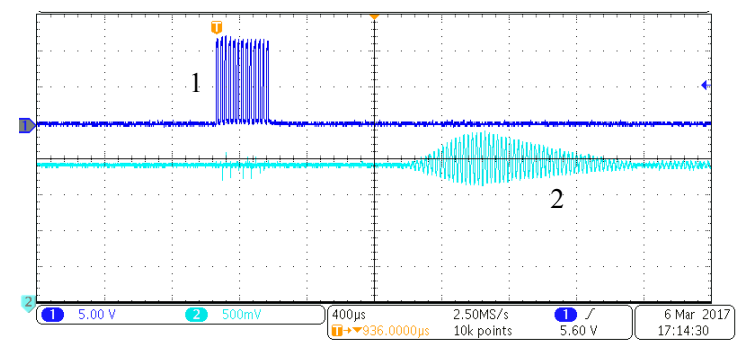

Figure 4. Transmitter excitation signal (dark, label 1) and received echo signal (light, label 2)

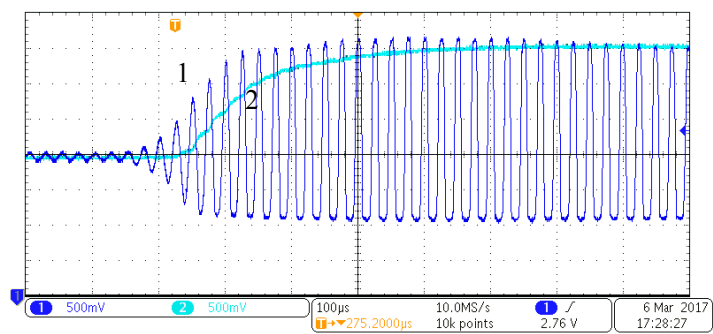

Figure 5. Amplified signal (dark, label 1) and its envelope (light, label 2)

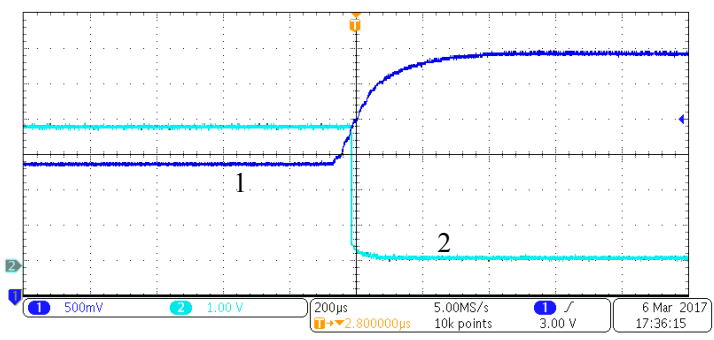

Figure 6. The envelope (dark, label 1) and comparator output (light, label 2)

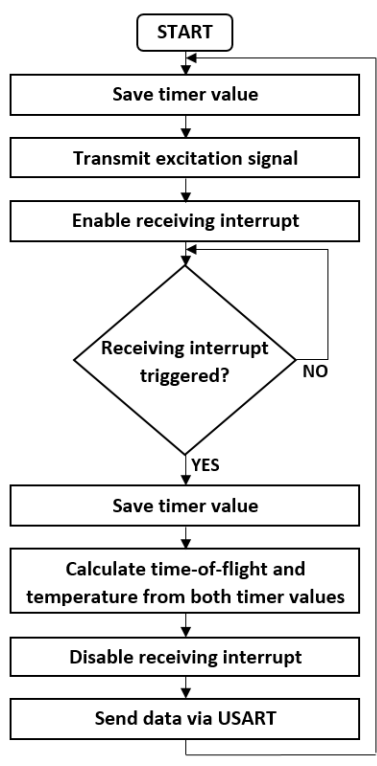

Figure 7. Software flowchart

\section{Measurement in stable temperature}

The prototype was tested in stable temperature to determine its measurement quality. Five series of short tests was performed with various duration (between 52 and $566 \mathrm{~s}$ ). Measurement repetition frequency $4 \mathrm{~Hz}$ was used in all measurements, resulting in 206-2263 measured temperature values. The 87 second measurement result is shown in the Fig. 8 and all measurements statistical results are summarized in the Table 1 . From the results we can state that $95 \%$ of the values are within $\pm 0.4{ }^{\circ} \mathrm{C}$ precision.

Averaging of the measured values is used in ultrasonic thermometry practice to filter out critical deviations and improve measurement precision. For example in [35], the average value from 50 measurements is used. The values after averaging from 10 measurements are shown in the Fig. 9 (in the case of third - i.e. $87 \mathrm{~s}$ - measurement series) and in the Table 2 (for all the measurement series). Small improvement of the precision can be observed, with 95 $\%$ of the values being within $\pm 0.3{ }^{\circ} \mathrm{C}$ precision.

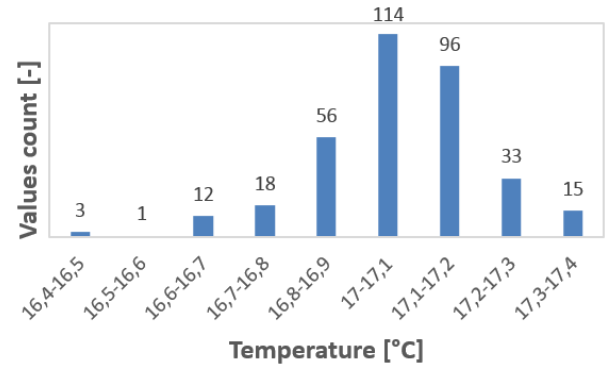

Figure 8. Histogram of third measurement series result in stable temperature 
TABLE I. STABLE TEMPERATURE MEASUREMENT RESULTS

\begin{tabular}{|c|c|c|c|c|c|c|}
\hline Series & $\begin{array}{c}\text { Duration } \\
{[\mathbf{s}]}\end{array}$ & $\begin{array}{c}\text { Values } \\
\text { count }\end{array}$ & $\begin{array}{c}\boldsymbol{\sigma} \\
{\left[{ }^{\circ} \mathbf{C}\right]}\end{array}$ & $\begin{array}{c}\text { Med } \\
{\left[{ }^{\circ} \mathbf{C}\right]}\end{array}$ & $\begin{array}{c}\text { Min } \\
{\left[{ }^{\circ} \mathbf{C}\right]}\end{array}$ & $\begin{array}{c}\text { Max } \\
{\left[{ }^{\circ} \mathbf{C}\right]}\end{array}$ \\
\hline 1 & 52 & 206 & 0.20 & 16.8 & 16.2 & 17.3 \\
\hline 3 & 55 & 219 & 0.21 & 17.0 & 16.3 & 17.3 \\
\hline 4 & 259 & 1036 & 0.17 & 17.1 & 16.2 & 17.6 \\
\hline 5 & 566 & 2263 & 0.21 & 16.9 & 16.0 & 17.4 \\
\hline
\end{tabular}

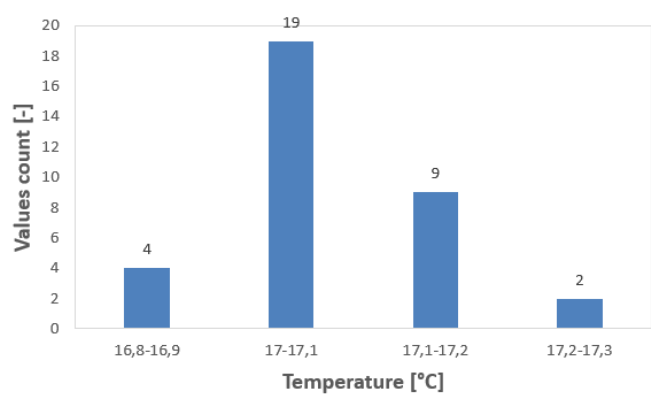

Figure 9. Histogram of third measurement series result in stable temperature after averaging (10pts)

TABLE II. STABLE TEMPERATURE MEASUREMENT RESULTS AFTER AVERAGING FROM 10 POINTS

\begin{tabular}{|c|c|c|c|c|c|c|}
\hline Series & $\begin{array}{c}\text { Duration } \\
{[\mathbf{s}]}\end{array}$ & $\begin{array}{c}\text { Values } \\
\text { count }\end{array}$ & $\begin{array}{c}\mathbf{\sigma} \\
{\left[{ }^{\circ} \mathbf{C}\right]}\end{array}$ & $\begin{array}{c}\text { Med } \\
{\left[{ }^{\circ} \mathbf{C}\right]}\end{array}$ & $\begin{array}{c}\text { Min } \\
{\left[{ }^{\circ} \mathbf{C}\right]}\end{array}$ & $\begin{array}{c}\text { Max } \\
{\left[{ }^{\circ} \mathbf{C}\right]}\end{array}$ \\
\hline 1 & 52 & 20 & 0.12 & 16.8 & 16.6 & 17.0 \\
\hline 3 & 55 & 21 & 0.16 & 17.0 & 16.7 & 17.2 \\
\hline 4 & 259 & 103 & 0.10 & 17.1 & 16.9 & 17.4 \\
\hline 5 & 566 & 226 & 0.15 & 16.9 & 16.6 & 17.2 \\
\hline
\end{tabular}

Measurement step of the prototype is $0.063{ }^{\circ} \mathrm{C}$ and measurement repetition frequency used was $4 \mathrm{~Hz}$.

\section{E. Measurement in thermal chamber}

An experiment in improvised thermal chamber was conducted to verify the thermometer prototype performance. The measurement was made in a thermal chamber in $28{ }^{\circ} \mathrm{C}-58{ }^{\circ} \mathrm{C}$ range. The results were compared to the values measured by a PT100 probe. Maximum prototype deviation from the PT100 values was $-2{ }^{\circ} \mathrm{C}$, with most measurements fitting in $\pm 1{ }^{\circ} \mathrm{C}$. This nonlinearity and precision does not reach the ultrasonic thermometry potential and is caused by the weaknesses coming from the usage of simple hardware and cheap material. On the other hand, simple hardware allows portability of the system and the achieved precision is sufficient for the measurement principle demonstration. Experiment results are shown in the Fig. 10 and Fig. 11.

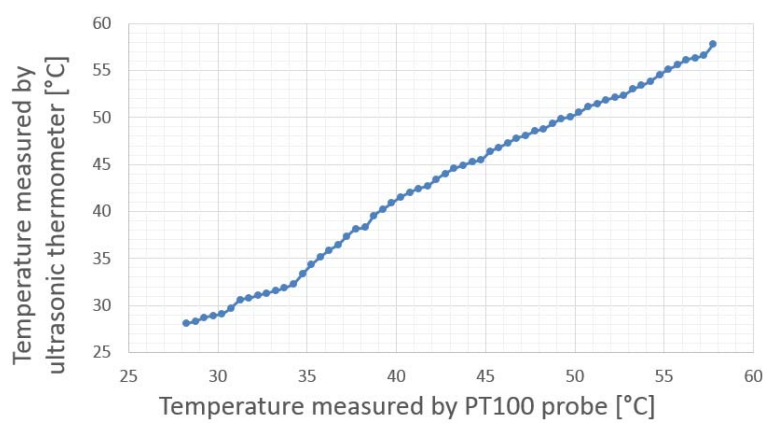

Figure 10. Experimental measurement results

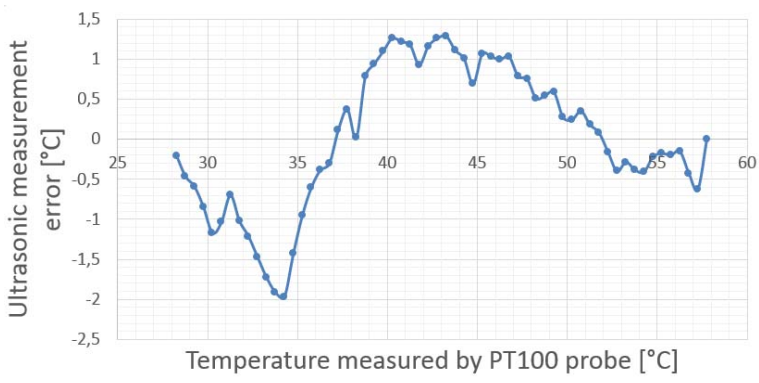

Figure 11. Experimental measurement errors

\section{CONCLUSION}

The state of the art study shows rich ultrasonic thermometry history along with many recent scientific studies. Various other interesting ultrasonic applications using similar principle as ultrasonic thermometry were mentioned. In conclusion, the ultrasonic thermometry is highly explored scientific area with new promising applications being discovered even in recent years and this year. Technology of this method is being still improved as well.

The ultrasonic temperature measurement prototype was developed in the experimental part of this paper. Advantage of being portable, simple to construct and usable for demonstration purposes on external sites comes with disadvantage of precision far from the potential of ultrasonic temperature measurement method. In stable temperature, raw measurement precision of $\pm 0.4{ }^{\circ} \mathrm{C}$ was achieved with the possibility to reach $\pm 0.3{ }^{\circ} \mathrm{C}$ by averaging of multiple vales. In temperature chamber measurement, $\pm 1{ }^{\circ} \mathrm{C}$ general precision with $-2{ }^{\circ} \mathrm{C}$ maximum deviation was observed. This result is sufficient for demonstrations and educational purposes, but for further studies and scientific work more precise system is needed. Authors plan to give up the simplicity of the used hardware and the portability of the prototype and implement more complex solution, which would be more perspective for their ongoing scientific goals in ultrasonic thermometry area.

\section{ACKNOWLEDGMENT}

Research described in the paper was supervised by doc. Ing. A. Platil, Ph.D., FEE CTU in Prague and supported by the Czech Technical University in Prague, under grant SGS16/170/OHK3/2T/13. 


\section{REFERENCES}

[1] E. Barrett, V. Suomi, "Preliminary report on temperature measurement by sonic means" J. Meteorol. 6, 1949, pp. 273276

[2] R. Schottland, "The measurement of wind velocity by sonic means" J. Meteorol. 12, 1955, pp. 386-390

[3] J. Kaimal, J. Businger, "A continuous wave sonic anemometer-thermometer" J. Appl. Meteorol. 2, 1962, pp. 156-164

[4] J. Kaimal, J. Businger, "Preliminary results obtained with a sonic anemometer-thermometer" J. Appl. Meteorol. 2, 1962, pp. $180-186$

[5] T. Hanafusa, T. Fujitani, Y. Kobori, Y. Mitsuna, "A new type of sonic anemometer-thermometer for field operation" Pap. Meteorol. Geophys. 33, 1982, pp. 1-19

[6] K. Arnold, A. Ziemann, A. Raabe, "Acoustic Tomography inside the Atmospheric Boundary Layer" Phys, Chem. Earth B 24, 1999, pp. 133-137

[7] K. Arnold, A. Ziemann, A. Raabe, "Near Surface Spatially Averaged Air Temperature and Wind Speed Determined by Acoustic Travel Time Tomography" Meteorologische Zeitschrift 10, 2001, pp. 61-70

[8] K. Arnold, A. Ziemann, A. Raabe, "Tomographic monitoring of wind and temperature in different heights above the ground” Acta Acust. United Acust. 87, 2001, pp. 703-708

[9] K. Arnold, A. Ziemann, A. Raabe, "Acoustic Tomography as a Remote Sensing Method to Investigate the Near-Surface Atmospheric Boundary Layer in Comparison with In Situ Measurement" Journal of Atmospheric and Oceanic Technology 19, 2002, pp. 1208-1215

[10] S. Vecherin, V. Ostashev, A. Ziemann, D. Wilson, K. Arnold, M. Barth, "Tomographic reconstruction of atmospheric turbulence with the use of time-dependent stochastic inversion" J. Acoust. Soc. Am. 122, 2007, pp. 1416-1425

[11] S. Vecherin, V. Ostashev, D. Wilson, A. Ziemann, “Timedependent stochastic inversion in acoustic tomography of the atmosphere with reciprocal sound transmission" Meas. Sci. Technol. 19, 2008, 125501 (12pp)

[12] S. Vecherin, V. Ostashev, D. Wilson, "Three-dimensional acoustic travel-time tomography of the atmosphere" Acta Acust United Acust. 94, 2008, pp. 349-358

[13] S. Vecherin, V. Ostashev, D. Wilson, A. Ziemann, G. Goedecke, "Recent Progress in Acoustic Travel-Time Tomography of the Atmospheric Surface Layer" Meteorologische Zeitschrift 18, 2009, pp. 125-133

[14] M. Barth, A. Raabe, "Accurate tomographic imaging of temperature and flow fields in air" Meas. Sci. Technol. 22, 2011, 035102 (13pp)

[15] F. Gueuning, M. Varlan, C. Eugene, P. Dupuis, "Accurate Distance Measurement by an autonomous Ultrasonic System Combining Time-of-Flight and Phase-Shift Methods" IEEE Transactions on Instrumentation and Measurement 46, 1997, pp. 1236-1240

[16] S. Huang, C. Huang, K. Huang, M. Young, "A high accuracy ultrasonic distance measurement system using binary frequency shift-keyed signal and phase detection" Review of Scientific Instruments, 2002, pp. 3671-3677

[17] K. Huang, C. Huang, Y. Li, M. Young, "High precision, fast ultrasonic thermometer based on measurement of the speed of sound in air" Review of Scientific Instruments, 2002, pp. 4022-4027

[18] C. Huang et al., "Design of an Ultrasonic System for Temperature Measurement in an Infant Incubator" 2003 IEEE EMBS Asian-Pacific Conference on Biomedical Engineering, 2003, pp. 296-297

[19] T. Liao W. Tsai, C. Huang, "A new ultrasonic temperature measurement system for air conditioners in automobiles" Meas. Sci. Technol. 15, 2004, pp. 413-419

[20] W. Tsai, H. Chen, T. Liao, "An ultrasonic air temperature measurement system with self-correction function for humidity" Meas. Sci. Technol. 16, 2005, pp. 548-555
[21] W. Tsai, H. Chen, T. Liao, "High accuracy ultrasonic air temperature measurement using multi-frequency continuous wave" Sensors and Actuators A 132, 2006, pp. 526-532

[22] Z. Huang, C. Qiao, Y. Wang, "Accurate ultrasonic measurement using MLS-modulated continuous waves" Measurement 60, 2015, pp. 178-185

[23] C. Zhou, Y. Wang, C. Qiao, S. Zhao, Z. Huang, "Highaccuracy ultrasonic temperature measurement based on MLSmodulated continuous wave" Measurement 88, 2016, pp. 1-8

[24] L. Lynnworth, E. Carnevale, M. McDonough, S. Fam, "Ultrasonic Thermometry for Nuclear Reactors" IEEE Transactions on Nuclear Science 16, 1969, pp. 184-187

[25] K. Koo, D. Jeong, J. Choi, D. Ko, “A New Measurement System of Very High Temperature in Atomic Pile using Ultrasonic Delay Time" IEEE International Conference on Electrical and Electronic Technology, 2001, pp. 860-863

[26] T. Chen, K. Nguyen, S. Wen, C. Jen, "Temperature measurement of polymer extrusion by ultrasonic techniques" Meas. Sci. Technol. 10, 1999, pp.139-145

[27] V. Putz, I. Burzic, J. Miethlinger, F. Maier, B. Zagar, "Adaptation of in-line ultrasonic velocimetry to melt flow measurement in polymer extrusion" Meas. Sci. Technol. 24, 2013, 107002 (6pp)

[28] M. Huger, D. Fargeot, C. Gault, "High-temperature measurement of ultrasonic wave velocity in refractory materials" High Temperatures - High Pressures 34, 2002, pp. 193-201

[29] T. Zalutskaya, I. Likhnovsky, Y. Lutsyk, “An Investigation of the Acoustic Properties of Materials for Ultrasonic Thermometry" Measurement Techniques 55, 2012, pp. 676680

[30] P. Schmidt, D. Walker, D. Yuhas, M. Mutton, "Thermal measurements using ultrasonic acoustical pyrometry" Ultrasonics 54, 2014, pp. 1029-1036

[31] Y. Wei et al., "Ultrasonic $\mathrm{Al}_{2} \mathrm{O}_{3}$ Ceramic Thermometry in High-Temperature Oxidation Environment” Sensors 16, 2016, 1905 (10 pp)

[32] I. Basarab-Horwath, M. Dorozhevets, "Measurement of the Temperature Distribution in Fluids Using Ultrasonic Tomography" 1994 IEEE Ultrasonics Symposium, 1994, pp. 1891-1894

[33] I. Jovanovic, L. Sbaiz, M. Vetterli, “Acoustic tomography for estimating temperature and wind flow" 13th International Symposium for the Advancement of Boundary Layer Remote Sensing, 2006, pp. 69-71

[34] S. Ohyama, J. Takayama, Y. Wanatabe, T. Takahoshi, K. Oshima, "Temperature Distribution and Wind vector Measurement Using CT Based on Time of Flight Detection" Sensors and Actuators A: Physical 151, 2009, pp. 159-167

[35] R. Jia et al., "Study of ultrasonic thermometry based on ultrasonic time-of-flight measurement" AIP Advances 6, 2016, 035006 (12pp)

[36] R. Jia, Q. Xiong, G. Xu, K. Wang, S. Liang, "A method for two-dimensional temperature field distribution reconstruction" Applied Thermal Engineering 111, 2017, pp. 961-967

[37] P. Richardson, M. Povey, "Ultrasonic temperature measurement and its potential for food processing systems" Food Control 1, 1990, pp. 54-57

[38] E. Sarabia, J. Llata, S. Robla, C. Torre-Ferrero, J. Oria, "Accurate Estimation of Airborne Ultrasonic Time-of-Flight for Overlapping Echoes" Sensors 2013, 13, 2013, pp. 1546515488

[39] I. Bazán, A. Ramos, A. Ramírez, L. Leija, "Ultrasonic thermometry inside tissues based on high-resolution detection of spectral shifts in overtones of scattering signals" Physics Procedia 63, 2015, pp. 158-162

[40] X. Zhao, J. Rose, "Ultrasonic guided wave tomography for ice detection" Ultrasonics 67, 2016, pp. 212-219

[41] O. Lufinka, "Ultrasonic Transceiver with the Possibilities of the Data Communication and the Two-Point Distance Measurement" 2016 International Conference on Applied Electronics, 2016 